\title{
A sitting or standing position - which one exerts more loads on the musculoskeletal system of the lumbar spine? Comparative tests based on the methods of mathematical modelling
}

\author{
HANNA ZADOŃ*, KATARZYNA NOWAKOWSKA-LIPIEC, ROBERT MICHNIK \\ Department of Biomechatronics, Faculty of Biomedical Engineering, Silesian University of Technology, Zabrze, Poland.
}

\begin{abstract}
Purpose: The work aimed to assess the functioning of the musculoskeletal system within the lumbar spine in relation to everyday postures of sitting and standing. Methods: The comparative analysis was based both on experimental tests and computer simulations performed in the AnyBody Modeling System environment. Input data used to prepare models were based on the information obtained in experimental tests. The test participants were tasked with adopting two postures: 1) standing position and 2) sitting position. Kinematics measurements were performed using the Zebris ultrasonic system. During sitting position, the tests additionally involved the use of a dynamometric platform measuring reaction forces occurring between buttocks and the seat. Results: The comparative analysis included measurements of the trunk inclination angle and the pelvic inclination angle as well as results of computer simulations. The sitting posture is responsible for increased trunk inclination and a change in the position of the pelvis. In terms of the sitting position, it was possible to observe an increase in the loads affecting individual intervertebral joints of the lumbar spine by $155-184 \%$ in comparison with the standing posture $(100 \%)$. Simulations revealed an increased muscle activity of the erector spinae, abdominal internal oblique muscles and abdominal external oblique muscles. Conclusions: Adopting a sitting position increases the loads on the lumbar spine and increases the activity of the erector spinae and abdominal muscles compared to the standing position, which is caused by change in the position of the pelvis and the curvature of the lumbar region.
\end{abstract}

Key words: sitting position, standing position, mathematical modeling, AnyBody Modeling System, lumbar spine, loads

\section{Introduction}

Learning at school, office work, commuting by car or spending one's free time in front of the TV made contemporary life dominated by the sitting posture. Statistical data show that, every day, an average European spends on average $300 \mathrm{~min}(180-420 \mathrm{~min})$ in the sitting position [14]. In spite of the fact that sitting does not require intense physical effort, remaining in the sitting posture for a long time adversely affects health, being responsible for postural defects and many other ailments. The information found in reference publications links the sedentary lifestyle to as many as
35 conditions including, among other things, back and musculoskeletal pain, hypertension, osteoporosis and cardiovascular disease [12].

The prevalence of the problem necessitates the verification of tests concerning the effect of the posture on the functioning of the musculoskeletal system. A survey carried out among 500 physiotherapists indicated a significant effect of education in terms of the optimum sitting and standing positions [11]. The issue of the optimum body posture has been discussed for decades. In 1947, the Posture Committee of the American Academy of Orthopaedic Surgeons described the posture as skeletomuscular balance, which supports structures of the body and protects against injury and

\footnotetext{
* Corresponding author: Hanna Zadoń, Department of Biomechatronics, Faculty of Biomedical Engineering, Silesian University of Technology, ul. Roosevelta 40, 41-800 Zabrze, Poland. Phone: +48 32277 74 36, e-mail: Hanna.Zadon@polsl.pl

Received: January 1st, 2021

Accepted for publication: January 18th, 2021
} 
deformity [11]. Positions (particularly long lasting ones) cause changes which adversely affect health, trigger pain and are responsible for the improper functioning of the locomotor system. Among other things, changes in the body posture include the protrusion of the head, the changes in spinal curvatures or the disturbance of a correct muscle balance [28], entailing numerous consequences in everyday life. Elderly persons tend to have increased thoracic kyphosis, affecting their gait patterns and, consequently, increasing the risk of falling [4].

The determination of the optimum body posture is not easy primarily because of differences between individuals, including base support, mass distribution and other anthropometric characteristics [4]. Authors of numerous reference publications attempted to identify the features of optimum standing or sitting positions, yet none of them indicated one ideal posture [11], [20]. In spite of the lack of scientific correlations linking a specific posture with pain, both in terms of the standing and sitting positions, physiotherapists consider the upright lordotic spinal posture as optimum for the functioning of the locomotor system [11], [20]. It should be remembered that opinions provided by physiotherapists are primarily based on knowledge connected with biomechanics, stereotypes concerning the optimum posture, own experience and the ability of linking body postures to disorders observed during appointment with patients. The improper and long-lasting loading of the locomotor system resulting from adopted postures can undoubtedly trigger pain [12]. An example of a method enabling the qualitative indication of loads exerted on the musculoskeletal system when adopting a specific position or a body posture is mathematical modelling with static optimisation. The possibilities of the method are presented in the remainder of the work.

The work aimed to compare the two most common postures adopted during the day, i.e., a standing and sitting position, with respect to loads transmitted by the musculoskeletal system.

\section{Materials and methods}

The comparison of the two basic postures of the day, i.e., standing and sitting, was performed on the basis of experimental tests and modelling.

The experimental tests were attended by 36 individuals (18 females $(\mathrm{F})$ and 18 males $(\mathrm{M})$ ) having an average weight of $72 \pm 17 \mathrm{~kg}(\mathrm{~F}=61 \pm 8 \mathrm{~kg}, \mathrm{M}=81 \pm$ $19 \mathrm{~kg})$ and an average height of $176 \pm 9 \mathrm{~cm}(\mathrm{~F}=170$ $\pm 6 \mathrm{~cm}, \mathrm{M}=183 \pm 7 \mathrm{~cm})$. The criteria for participation in the experiments were the lack of pain in the lower spine, the lack of visible faulty posture and no previous surgeries in the lumbo-pelvic-hip complex and the lower limbs. The test participants were tasked with adopting two postures: 1) standing position with arms along the trunk and 2) sitting position (without the backrest) - a natural sitting position with the knees bent at a right angle in the knee joint. Measurements of the adopted postures which were subjected to analysis involved the use of the Zebris ultrasonic system (Zebris Medical GmbH, Germany). This enabled an objective assessment of characteristic anthropometric points in space and positions of body segments in relation to one another. The sitting posture involved additional measurements of reaction forces occurring between the seat and the buttocks and ground reaction forces. The aforesaid measurements involved the use of a Zebris FDM-S dynamometric platform placed on the seat (Zebris Medical GmbH, Germany) and Medilogic Insoles - foot pressure measuring system ( $\mathrm{T} \& \mathrm{~T}$ Medilogic Medizintechnik GmbH, Germany).

The results of kinematics-related tests (trunk inclination angle and pelvic inclination angle) as well as the results of tests concerning seat and ground reaction forces constituted input data for simulations performed in the AnyBody Modeling System environment (AnyBody Technology Inc., Aalborg, Denmark). The standing and sitting postures of the test participants were modelled using mathematical models of the human musculoskeletal system available in the AMMR repository (Fig. 1). The simulations of the standing position were performed using the model of the entire human body (StandingModel), whereas the simulations of the sitting position involved the use of the FreePostureModel. Models in the AnyBody environment are composed of 69 rigid bodies presenting the skeletal system and approximately 1000 linear elements representing the muscular system [7]. The developed models used the default lumbar spine rhythm, which, on the basis of reference publications, [27] determines the range of movements at individual levels of the lumbar spine. The adopted rhythm is characterised by the increased participation of single vertebrae in the movement of the entire lumbar spine along with the transition from the lower spine upwards. The models involved the use of the ScalingLengthMassFat method, which, based on the information about height, body mass and the percentage content of the fatty tissue (calculated using BodyMass Index) of a given person, scaled the dimensions of body segments and muscular forces. The input data, which was used to develop the models of activities 
subjected to analysis, included information about the body mass and height of a given test participant, the value of the trunk inclination angle and that of the pelvic inclination angle and, regarding the sitting posture, information about reaction forces occurring between the buttocks and the seat and ground reaction forces.

The development of mathematical models of the standing and sitting positions involved the adoption of the following assumptions and simplifications:

- symmetricity of the human body structure and the symmetricity of the adopted static posture;

- muscular model - AnyMuscleModel - a simple model assuming the constant value of muscular force independent of its working conditions;

- posture during the adoption of the standing and sitting positions was modelled solely on the basis of data concerning the trunk inclination angle (defined as the angle between the straight line determined by $\mathrm{C} 7$ and L5/S1 and the vertical straight line) and the pelvic inclination angle in the sagittal plane (defined as the angle between the straight line determined by the anterior superior iliac spines and posterior superior iliac spines and the horizontal straight line) - Fig. 1c;

- seat reaction force was modelled using two vectors of forces applied appropriately in the pubic bone area, whereas the value of the vectors was averaged from the results recorded on the dynamometric platform during a 10 -second measurement;

- values of ground reaction forces in relation to the standing position were determined using an algorithm incorporated into the StandingModel. In relation to the sitting position, the above-named values were modelled using two vectors of forces applied in the area of the heel and that of the instep.

The determination of loads affecting the musculoskeletal system in the AnyBody Modeling System environment was obtained through solving an inverse dynamic problem; the identification of muscular forces was obtained by using static optimisation. The optimisation criterion was the criterion of movement control assuming the minimisation of the sum of the cube power of the proportion of muscular forces to their maximum values [7], [10], [15].

The comparative analysis encompassed the trunk inclination angle and the pelvic inclination angle as well as the results of computer simulations, the resultant values of reaction forces in the intervertebral joints of the lumbar spine and muscular forces present in the lumbar spine when adopting the standing and sitting postures. The simulation results were standardised in relation to $(\mathrm{BW})$. The results were also analysed in relation to sex. Normalcy of the distribution of the analysed variables was verified using the Shapiro-Wilk test, in which the significance level was set at $\alpha=0.05$. The correlation between the parameters subjected to analysis was examined using the Pearson correlation; the level of statistical significance of the correlation coefficient being $p<0.05$.

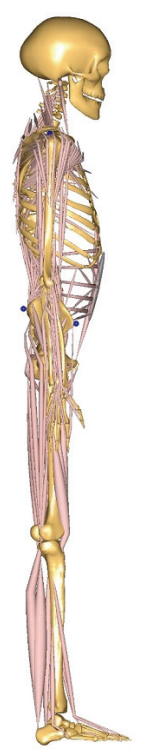

(a)

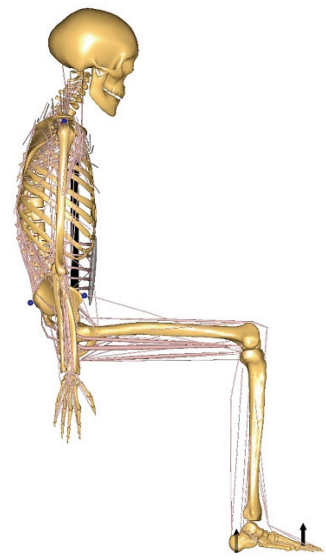

(b)

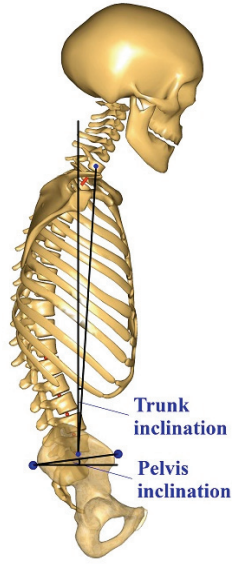

(c)
Fig. 1. Examples of: a) the standing position, b) the sitting position modelled in the AnyBody Modeling System environment, c) determining the torso and pelvic angle inclination

\section{Results}

The experimental tests and computer simulations made it possible to perform the comparative analysis of the effect of the standing and sitting positions on loads exerted on the lumbar spine.

In Table 1, kinematic data, i.e., the trunk inclination angle and the pelvic inclination angle recorded during the standing and sitting positions are presented. In relation to the entire group, the average trunk inclination during the standing position amounted to $2.34^{\circ} \pm 1.88^{\circ}$. After dividing the group according to sex, it was observed that the trunk inclination angle in the group of female participants was slightly smaller $\left(2.23^{\circ} \pm 1.80^{\circ}\right)$ than in the group of males $\left(2.45^{\circ} \pm 1.96^{\circ}\right)$. The comparison of the standing posture with the sitting posture revealed that the adoption of the sitting position was responsible for a nearly 3 -fold increase in the trunk inclination, where its mean value amounted to $7.00^{\circ} \pm 3.59^{\circ}$. The sitting posture-related difference was slightly higher in relation to sex. In the group of males, the value 
of trunk inclination angle $\left(7.38^{\circ} \pm 3.62^{\circ}\right)$ was by $0.69^{\circ}$ higher than that in the group of women $\left(6.63^{\circ} \pm 3.53^{\circ}\right)$. A visible difference according to sex was also observed in relation to the pelvic position. In the standing position, in all of the test participants the pelvis was in anteversion (the mean pelvic inclination angle being $-16.09^{\circ} \pm$ $\left.4.99^{\circ}\right)$. In the group of females, the mean pelvic inclination angle amounted to $-17.48^{\circ} \pm 4.45^{\circ}$, whereas in the group of males, the mean pelvic inclination angle amounted to $-14.62^{\circ} \pm 5.11^{\circ}$. During the sitting position, the pelvis was in retroposture in $73 \%$ and in anteversion in $27 \%$ of the test participants ( $70 \%$ being females). The pelvic inclination angle in the sagittal plane was restricted within the range of $-25.43^{\circ} \div 7.90^{\circ}$. The higher inclination values recorded in the sitting posture indicate greater differences in kinematics in the sitting posture than in the standing one.

The analysis of the results of the simulation revealed that the adoption of the sitting position significantly increased loads present in the lumbar spine - Fig. 2. In comparison with the standing position, the sitting posture is responsible for an increase in the resultant reaction force in individual segments of the lumbar spine, i.e., by $55 \%$ in relation to segment L5-S1 (an increase from $0.7 \pm 0.05 \mathrm{BW}$ to $1.08 \pm 0.17 \mathrm{BW}$ ), $67 \%$ - L4-L5 (an increase from $0.61 \pm 0.05 \mathrm{BW}$ to $1.02 \pm 0.17 \mathrm{BW}$ ), 78\% - L3-L4 (an increase from $0.58 \pm 0.05 \mathrm{BW}$ to $1.02 \pm 0.17 \mathrm{BW}), 78 \%-\mathrm{L} 2-\mathrm{L} 3$ (an increase from $0.60 \pm 0.06 \mathrm{BW}$ to $1.05 \pm 0.16$ ), $84 \%$ - L2-L3 (an increase from $0.63 \pm 0.06 \mathrm{BW}$ to $1.14 \pm 0.17 \mathrm{BW}$ ) and $80 \%$ (an increase from 0.65 $\pm 0.06 \mathrm{BW}$ to $1.13 \pm 0.18 \mathrm{BW}$ ) - Fig. 1a. The observations of the results according to sex (Fig. 1b) revealed that, in terms of the sitting position, the values of the resultant reaction forces were by between $6 \%(\mathrm{~L} 1-\mathrm{L} 2)$ and up to $16 \%$ (L5-S1) higher in relation to the group of males (Fig. 1b), which could be ascribed to a more common pelvic retroposture and greater trunk inclination in this group. The analysis of correlations revealed the existence of a moderately strong relation between the resultant reaction force in individual intervertebral joints of the lumbar spine and the trunk inclination angle $(r=0.53 \div 0.70 ; p<0.05)$ as well as between the resultant reaction force in intervertebral joints of the lumbar spine and the pelvic inclination angle $(r=$ $0.46 \div 0.66 ; p<0,05)$ in the sitting position.

In Figure 3, presents the values of muscular forces present in the lumbar spine - erector spinae and abdomi-

Table 1. Trunk inclination angle and pelvic inclination angle in relation to the standing and sitting position (pelvic inclination angle - designations: $(-)$ anteversion, $(+)$ retroposture)

\begin{tabular}{|c|c|c|c|c|c|}
\hline & & & Total group $\left[^{\circ}\right]$ & Women $\left[{ }^{\circ}\right]$ & $\operatorname{Men}\left[{ }^{\circ}\right]$ \\
\hline \multirow{4}{*}{ 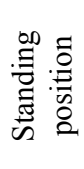 } & \multirow{2}{*}{ Trunk inclination [deg] } & Mean \pm SD & $2.33 \pm 1.88$ & $2.22 \pm 1.80$ & $2.44 \pm 1.95$ \\
\hline & & Range & $0.00 \div 6.80$ & $0.10 \div 6.30$ & $0.00 \div 6.80$ \\
\hline & \multirow{2}{*}{ Pelvis inclination [deg] } & Mean \pm SD & $-16.09 \pm 4.99$ & $-17.48 \pm 4.45$ & $-14.62 \pm 5.11$ \\
\hline & & Range & $-25.43 \div-5.36$ & $-24.70 \div-7.90$ & $-25.43 \div-5.36$ \\
\hline \multirow{4}{*}{ 点: } & \multirow{2}{*}{ Trunk inclination [deg] } & Mean \pm SD & $6.99 \pm 3.59$ & $6.63 \pm 3.53$ & $7.38 \pm 3.62$ \\
\hline & & Range & $0.30 \div 15.30$ & $0.30 \div 15.30$ & $0.61 \div 12.80$ \\
\hline & \multirow{2}{*}{ Pelvis inclination [deg] } & Mean \pm SD & $4.76 \pm 8.09$ & $2.94 \pm 9.43$ & $6.69 \pm 5.76$ \\
\hline & & Range & $-11.26 \div 25.41$ & $-11.26 \div 25.41$ & $-5.26 \div 15.84$ \\
\hline
\end{tabular}

Lumbar spine

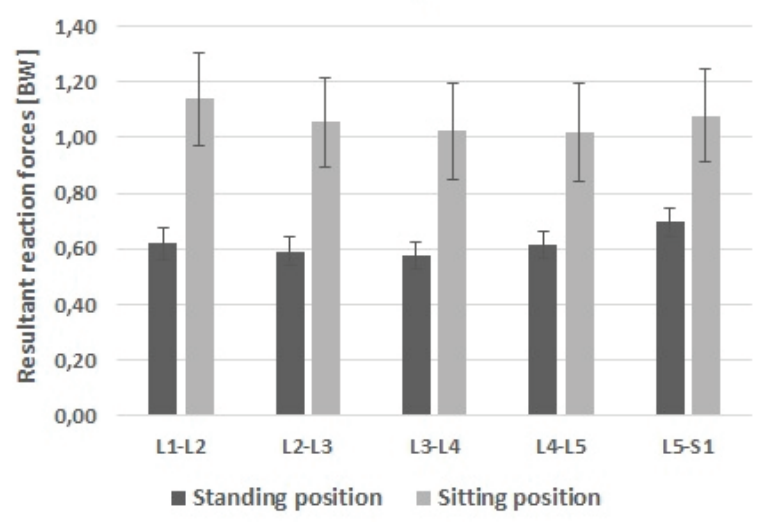

L5-S1 spinal segment

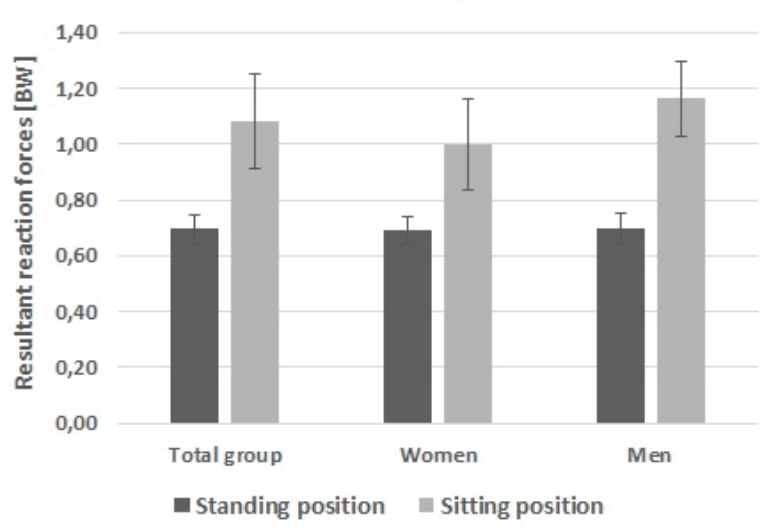

Fig. 2. Resultant reaction forces in the intervertebral joints of the lumbar spine 

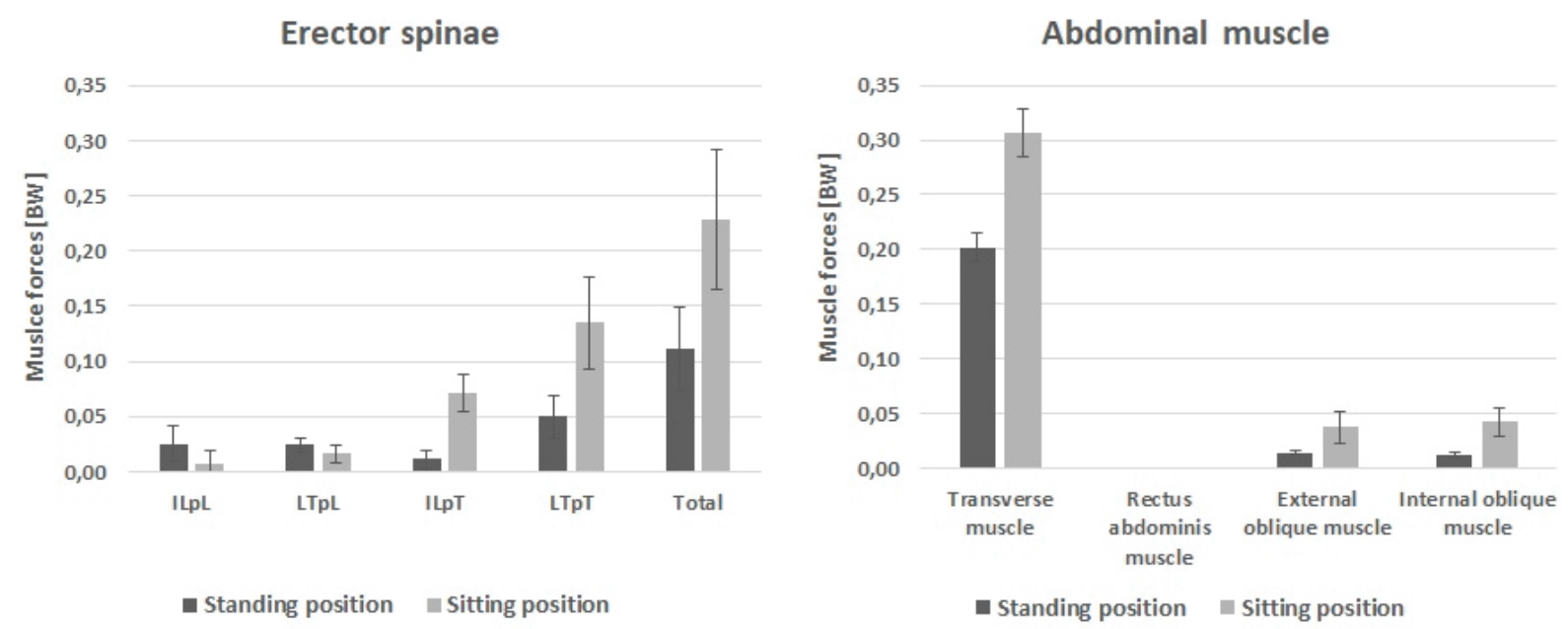

Fig. 3. Muscular forces generated by the spine muscles present in the lumbar spine

nal muscles - transversus abdominis, rectus abdominis, abdominal internal oblique muscle and abdominal external oblique muscle. The analysis of the erector spinae involved division into four groups, i.e., ILpL (lat. iliocostalis lumborum pars lumborum), ILpT (lat. Iliocostalis lumborum pars thoracis), LTpL (lat. longissimus thoracis pars lumborum) and LTpT (lat. Longissimus thoracis pars thoracis). Among the muscles subjected to analysis, the highest activity was that of the transversus abdominis, in relation to which the muscular force during the standing position amounted to $0.20 \pm$ $0.01 \mathrm{BW}$, whereas during the sitting position it was by $50 \%$ higher and amounted to $0.31 \pm 0.02 \mathrm{BW}$. The remaining muscles, i.e., the abdominal internal and external oblique muscles were characterised by similar activity (standing posture: $0.01 \pm 0.0 \mathrm{BW}$ and sitting posture: $0.04 \pm 0.01 \mathrm{BW}$ ). In turn, the activity of the rectus $a b$ dominis was low as the value of muscular force was close to zero. Similarly to abdominal muscles, a higher total activity of the erector spinae was recorded in the sitting position, i.e., $0.23 \pm 0.06 \mathrm{BW}$, than in the standing position, i.e., $0.11 \pm 0.04 \mathrm{BW}$. Differences in the muscular forces generated by the erector spinae in relation to a given posture were observed during the analysis of its individual muscular groups. During standing, higher activity was noticed in relation to groups ILpL and LTpL, whereas during sitting, higher activity was observed in relation to groups ILpTand LTpT.

\section{Discussion}

Presently, most views on the optimum sitting and standing postures result from physiotherapists' opinions based on their knowledge, experience and, most frequently, the association of lower spine pain with the posture of patients [11], [20]. The presented method of experimental tests and computer-aided simulations enable the quantitative assessment of loads occurring within the locomotor system. The measurements of kinematics and external forces and their use in the development of mathematical models constitute a tool which enables a complex analysis related to the effect of an adopted posture on the values of loads exerted on the musculoskeletal system. This work contains the comparison of the two basic postures adopted in everyday life, i.e., the standing and sitting positions.

\section{Kinematics}

The standing position and the sitting position undoubtedly differ in terms of kinematics. Data obtained in the tests as well as information presented by Philippot et al. [22] and Claeys et al. [4] unquestionably indicate different positions of the pelvis. Both the authors of this work and Philippot et al. [22] observed during the sitting position a tendency to change the pelvic inclination angle by approximately $20^{\circ}$ in relation to the standing posture. The change results in a smaller anteversion or retroposture if compared with that characteristic of the standing posture. Numerous reference data confirm the effect of the pelvic inclination angle on the sagittal posture of the spine in its lower parts, i.e., in the lumbosacral section [2], [8], [15]. In more than $73 \%$ of the test participants, the pelvic retroposture during the position of sitting was responsible for the loss of the natural curvature of the lumbar spine, i.e., the flattening of the lumbar lordosis [2], [15]. The results of measurements presented by Lord et al. [13] indicate that the angle of the lumbar lordosis in the standing posture is on average greater by $50 \%$ than during the sitting position. During the analysis of 
the lumbo-pelvic-hip complex, it is also necessary to pay attention to sex-related differences which, during the tests discussed in this work, were observed both in the standing and sitting posture. Janssen et al. [9] confirmed that males and females have a different sagittal spino-pelvic alignment, which results from varying anatomical structures. The adoption of both standing and sitting positions was also affected by obesity [1], [16]. Buckland et al. [1] observed that sitting individuals with BMI $>25$ were characterised by increased pelvic retroposture, the aim of which was the compensation for soft-tissue impingement that occurs anterior to the hip joint and limits hip flexion. Apart from the influence of mass, Mieszała et al. [16] also indicated age and body height as significant determinants of the size of the pelvic tilt angle. In the tests discussed in the article the value of BMI was below 25. Because of the foregoing, the simulation results should be generalised only in relation to the population without obesity.

\section{Loads exerted on the musculoskeletal system of the lumbar spine in the standing and sitting positions}

Simulations performed in the AnyBody Modeling System environment enabled the determination of reaction forces in the intervertebral joints and muscular forces present in the lumbar spine.

The results presented in the work in accordance with the overview of previously performed tests of intradiscal pressure (IDP) in the lumbar spine revealed higher loads in the sitting position than in the standing position [5]. Nachemson et al. [17] demonstrated that pressure in the intervertebral disk during sitting without the backrest is by as many as $40 \%$ higher than that during the standing position. In turn, leaning forwards by $20^{\circ}$ increases the pressure to $190 \%$. Thirty years later, Sato et al. [25] also confirmed that the adoption of the sitting posture may increase pressure up to approximately $210 \%$ in relation to the sitting posture with the trunk inclination, $115 \%$ in relation to the sitting upright posture and $137 \%$ in relation to the sitting position hyperextended in relation to the standing position (100\%). Surprisingly, Wilke et al. [26], in relation to the natural sitting posture, recorded the value of IDP similar or even slightly lower than in the sitting position and also recorded pressure increased up to approximately $165 \%$ in the inclined sitting posture. In this work, the adoption of the sitting position increased loads in the individual segments of the lumbar spine between $155 \%$ and even $184 \%$ in comparison with the standing posture $(100 \%)$. The correlation results revealed that the increase in the resultant reaction force in the intervertebral joints of the lumbar spine was affected both by the position of the pelvis (increased retroposture generates higher loads [15]) and increased trunk inclination, which was also observed in works by Rohlmann et al. [24] and Nowakowska et al. [19]. The comparison of the simulation results with the data found in reference publications [5], [17], [19], [24][26] revealed that values of loads were comparable, which confirmed the correctness of the assumptions adopted during modelling. Slight differences between values could result from the different positioning of individual body segments in the sitting posture as well as from a small number of test participants in groups (Nachemson -9 participants, Sato -8 participants and Wilke - 1 participant). The application of mathematical modelling made it possible to perform the analysis of loads in relation to 36 persons, which enabled the analysis of 36 various standing positions and 36 various sitting positions.

The adoption of a standing or sitting position also leads to differences in the activity of the muscular system. The simulations revealed that, in comparison with standing, the sitting posture is characterised by increased values of muscular forces generated by the erector spinae, transversus abdominis as well as abdominal internal and external oblique muscles. Information available in reference publications concerning muscular activity during the standing and sitting positions are based on EMG [6], [21] and USG [23] measurements. However, there are no data concerning values of muscular forces. In addition, it should be noticed that EMG and USG measurements enable the analysis of large muscle groups located directly under the skin surface, whereas the use of mathematical modelling makes it possible to determine muscular activity and muscular forces of all modelled muscles. O'Sullivan et al. [21] demonstrated a change in muscular activity depending on the adopted, both standing and sitting, positions - in the standing and sitting postures, the superficial thoracic erector spinae muscle and the anteroinferior portion of the internal oblique muscle are often activated, and they have less activation in passive trunk postures. In turn, comparing the sitting and standing positions, similar to this work, a greater muscular activity of the thoracic erector spinae muscle was observed during the sitting position. However, different correlations were obtained in relation to the muscular activity of internal oblique muscle O'Sullivan et al. recorded a lower muscular activity during the sitting position than during the standing posture. Claus et al. [6] analysed 4 sitting postures characterised by subtle changes in the thoracolumbar and lumbar spinal curves. The results revealed varying 
magnitudes of muscle activity in relation to postures, particularly in the deep and superficial regions of the lumbar multifidus. In turn, USG measurement results presented by Reeve et al. [23] confirmed the effect of the adopted posture on the thickness of the transversus abdominis - in both erect lumbo-pelvic neutral standing and sitting postures there was an increase in the transversus abdominis thickness compared to swayback standing or slouched sitting, respectively. The comparison of the erect standing posture with the erect sitting posture revealed slightly greater thickness of the transversus abdominis during the position of standing. The authors suggested that the increased thickness of muscle indicates increased activity. However, the aforesaid thesis was not confirmed in the research performed by the authors of this article as the simulation results indicated the increased activity of the transversus abdominis in the sitting position.

Information contained in reference publications confirmed that the activity of trunk muscles depends on the adopted sitting position, which may vary significantly. Different positions of individual segments of the body during the position of sitting may trigger various activity of individual muscles. The aforesaid differences are discussed in the above-named reference publications. It should also be noted that, during the tests, the manner of sitting was not imposed - test participants were tasked with adopting a neutral posture. The work also presents results in relation to the averaged neutral sitting position.

\subsection{Limitation of this work and directions of further research}

The presented research work has some limitations related to the applied method. Both the standing and sitting postures were modelled solely on the basis of information about the trunk inclination angle and pelvic inclination angle. The lordosis of the lumbar spine was determined using an algorithm describing the lumbopelvic rhythm implemented in the AnyBody Modeling System environment [27]. Because of the previously assumed limitations in both the StandingModel and the FreePostureModel, simulations of individual postures did not take into account the changes in thoracis kyphosis. Another limitation was not taking into consideration ligaments and passive properties of intervertebral discs in the model. However, on the basis of the information contained in available reference publications [3], [18], the authors suppose that the aforesaid limitations should not significantly affect the findings resulting from the analy- ses. Another simplification which was applied to the tests was the modelling of the seat reaction force by means of two 2 vectors.

The experimental tests and simulations as well as the analysis of the related results indicate the necessity of continuing the research in several areas. In subsequent research-related works, the authors intend to develop a model of the sitting posture taking into account individual spine curvatures measured during experimental tests. Such a model will make it possible to reflect, in a more precise way, the distribution of pressure forces measured using the dynamometric platform.

\section{Conclusions}

The above-presented experimental tests and modelling made it possible to compare the position of individual segments of the body and the loads exerted on the musculoskeletal system during the standing and sitting posture. The results-based analysis justified the formulation of the following conclusions:

- sitting posture forces a change in the position of the pelvis by on average $21.21^{\circ} \pm 7.44^{\circ}$ in relation to the standing posture; the pelvic anteversion or retroposition is smaller than that of the standing posture, thus reducing the angle of lordosis;

- standing position does not increase muscle strength, but only stimulates individual muscles or activates the strength necessary to maintain body position, whereas sitting position increases the muscular force of the erector spinae, transversus abdominis, abdominal internal oblique and abdominal external oblique muscles;

- sitting posture may on average increase loads in individual segments of the lumbar spine by $155-184 \%$ in relation to the standing posture $(100 \%)$;

- it was demonstrated that, during the sitting position, loads (resultant reaction forces in the intervertebral joints of the lumbar spine) affected the trunk inclination angle and pelvic inclination angle:

pelvic retroposition was responsible for increased loads,

the higher the trunk inclination, the greater the loads.

\section{Acknowledgements}

Publication supported by Own Schoolarship Fund of the Silesian University of Technology in year 2019/2020, grant number: 32/FSW18/0003-03/2019. 


\section{Conflicts of interest}

The authors declare no conflict of interest.

\section{References}

[1] Buckland A.J., Burapachaisri A., Stekas N., Vasquez-Montes D., Protopsaltis T., VigdorchiK J., Obesity Alters Spinopelvic Alignment Changes From Standing to Relaxed Sitting: the Influence of the Soft-tissue Envelope, Arthroplast. Today, 2020, 6, 590-595.e1.

[2] De Carvalho D.E., Soave D., Ross K., Callaghan J.P., Lumbar Spine and Pelvic Posture Between Standing and Sitting: A Radiologic Investigation Including Reliability and Repeatability of the Lumbar Lordosis Measure, J. Manipulative Physiol. Ther., 2010, 33, 48-55.

[3] Cholewicki J., McGill S.M., Lumbar posterior ligament involvement during extremely heavy lifts estimated from fluoroscopic measurements, J. Biomech., 1992, 25, 17-28

[4] Claeys K., Brumagne S., Deklerck J., Vanderhaeghen J., DANKAERTS W., Sagittal evaluation of usual standing and sitting spinal posture, J. Bodyw. Mov. Ther., 2016, 20, 326-333.

[5] Claus A., Hides J., Moseley G.L., Hodges P., Sitting versus standing: Does the intradiscal pressure cause disc degeneration or low back pain?, J. Electromyogr. Kinesiol., 2008, $18,550-558$.

[6] Claus A.P., Hides J.A., Moseley G.L., Hodges P.W., Different ways to balance the spine: Subtle changes in sagittal spinal curves affect regional muscle activity, Spine (Phila. Pa. 1976), 2009, 34, E208-E214.

[7] Damsgaard M., Rasmussen J., Christensen S.T., Surma E., DE ZEE M., Analysis of musculoskeletal systems in the AnyBody Modeling System, Simul. Model. Pract. Theory, 2006, $14,1100-1111$.

[8] Hayden A.M., Hayes A.M., Brechbuhler J.L., IsRael H., PlaCE H.M., The effect of pelvic motion on spinopelvic parameters, Spine J., 2018, 18, 173-178.

[9] Janssen M.M.A., Drevelle X., Humbert L., Skalli W., CASTELEIN R.M., Differences in male and female spino-pelvic alignment in asymptomatic young adults: A three-dimensional analysis using upright low-dose digital biplanar X-rays, Spine (Phila. Pa. 1976)., 2009, 34, E826-E832.

[10] Koblauch H., Low back load in airport baggage handlers, Dissertation, Univeristy of Copenhagen, Denmark, 2015.

[11] Korakakis V., O'Sullivan K., O'Sullivan P.B., Evagelinou V., Sotiralis Y., Sideris A., SaKellariou K., Karanasios S., Giakas G., Physiotherapist perceptions of optimal sitting and standing posture, Musculoskelet. Sci. Pract., 2019, 39, 24-31.

[12] LEVINE J.A., Sick of sitting, Diabetologia, 2015, 58, 1751-1758.

[13] LORD M.J., SMALl J.M., DinSAY J.M., WATKInS R.G., Lumbar lordosis: Effects of sitting and standing, Spine (Phila. Pa. 1976), 1997, 22, 2571-2574.

[14] Loyen A., Van Der Ploeg H.P., Bauman A., Brug J., LAKERVELD J., European sitting championship: Prevalence and correlates of self-reported sitting time in the 28 European Union Member States, PLoS One, 2016, 11, e0149320.
[15] MichniK R., ZadoŃ H., NowakOwSKA-LipieC K., JOCHMYCZYK-WoźNIAK K., MYŚLiWIEC A., MitAs A.W., The effect of the pelvis position in the sagittal plane on loads in the human musculoskeletal system, Acta Bioeng. Biomech., 2020, 22, 33-42.

[16] Mieszala W., Demczuk-WŁodarczyk E., Chromik K., HaWrylaK A., MaŁachowsKa-SobiESKa M., Examining relationships of the anterior pelvic tilt angle with the anteriorposterior curvatures and elongation of the spine, Acta Bioeng. Biomech., 2019, 21, 37-44.

[17] NACHemson A., ElfSTRÖM G., Intravital dynamic pressure measurements in lumbar discs. A study of common movements, maneuvers and exercises., Scand. J. Rehabil. Med. Suppl., 1970, 1, 1-40.

[18] NowakowSKa-LiPIEC K., Michnik R., LineK P., MYŚLIWIEC A., JOCHYMCZYK-WOźNIAK K., GzIK M., A numerical study to determine the effect of strengthening and weakening of the transversus abdominis muscle on lumbar spine loads, Comput. Methods Biomech. Biomed. Engin., 2020, 1-10, DOI: 10.1080/10255842.2020.1795840.

[19] Nowakowska K., Gzik M., Michnik R., Myśliwiec A., JurkojĆ J., SuchoŃ S., BuRKaCKi M., The Loads Acting on Lumbar Spine During Sitting Down and Standing Up, [in:] M. Gzik, E. Tkacz, Z. Paszenda, E. Piętka (Eds.), Innovations in Biomedical Engineering. Advances in Intelligent Systems and Computing, Springer, Cham, 2017, 169-176.

[20] O'Sullivan K., O'Sullivan P., O’Sullivan L., DanKaerts W., What do physiotherapists consider to be the best sitting spinal posture?, Man. Ther., 2012, 17, 432-437.

[21] O'Sullivan P.B., Grahamslaw K.M., Kendell M., LAPENSKIE S.C., MÖLler N.E., Richards K. V., The effect of different standing and sitting postures on trunk muscle activity in a pain-free population, Spine (Phila. Pa. 1976), 2002, 27, 1238-1244.

[22] Philippot R., Wegrzyn J., Farizon F., Fessy M.H., Pelvic balance in sagittal and Lewinnek reference planes in the standing, supine and sitting positions, Orthop. Traumatol. Surg. Res., 2009, 95, 70-76.

[23] ReEve A., Dilley A., Effects of posture on the thickness of transversus abdominis in pain-free subjects, Man. Ther., 2009, 14, 679-684.

[24] Rohlmann A., Zander T., Graichen F., Dreischarf M., BERGMANN G., Measured loads on a vertebral body replacement during sitting, Spine J., 2011, 11, 870-875.

[25] Sato K., KIKUChi S., Yonezawa T., In vivo intradiscal pressure measurement in healthy individuals and in patients with ongoing back problems, Spine (Phila. Pa. 1976), 1999, 24, 2468-2474.

[26] Wilke H., Neef P., Caimi M., Hoogland T., Claes L.E., New In Vivo Measurements of Pressures in the Intervertebral Disc in Daily Life, Spine (Phila. Pa. 1976), 1999, 24, 755-762.

[27] Wong K.W.N., LuK K.D.K., LeONG J.C.Y., Wong S.F., Wong K.K.Y., Continuous Dynamic Spinal Motion Analysis, Spine (Phila. Pa. 1976), 2006, 31, 414-419.

[28] Zyznawska J., Frankowski G., Wodka-Natkaniec E., KolomańSKA D., Boczoń K., KulesA-MrowieckA M., Disbalance and fatigue of the spinal extensors as one of the causes of the overload disease of the lumbar spine, Acta Bioeng. Biomech., 2019, 21, 119-125. 